\title{
Effect of repeated ischaemic preconditioning on TLR4 and proinflammatory cytokines TNF- $\alpha$ and IL-1 $\beta$ in myocardial ischaemia-reperfusion injury in a rat model
}

Ma Yu ${ }^{1}$, Ni Wen ${ }^{1}$, Zhu Wenzhong ${ }^{1}$, Xiong Yuanchang ${ }^{1}$, Deng Xiaoming ${ }^{1}$, Luo Yongjin ${ }^{2}$

1Department of Anesthesia, Changhai Hospital, Second Military Medical University, Shanghai, China

2Department of Cerebral Surgery, Changhai Hospital, Chongqing Medical University, Chongqing, China

Submitted: 27 August 2010

Accepted: 5 October 2010

Arch Med Sci 2010; 6, 6: 843-847

DOI: 10.5114 /aoms.2010.19289

Copyright $\odot 2010$ Termedia \& Banach

\section{Abstract}

Introduction: The role of TLR4 in ischaemic preconditioning is still unclear; we do not know the change of the expression of TLR4 in the process. In this study, we used ischaemic preconditioning models to observe the change of TLR4 expression and the level of proinflammatory cytokines TNF- $\alpha$ and IL-1 $\beta$ to investigate the protective mechanism of TLR4 in ischaemic preconditioning for myocardial ischaemia-reperfusion injury (MI/RI) in rats.

Material and methods: Eighteen male Sprague-Dawley (SD) rats were randomly separated into sham, ischaemic reperfusion (IR) and ischaemic preconditioning (IP) groups (6/group). Peripheral blood and cardiac muscle with pathological changes were collected after the establishment of the above three animal models. We used ELISA to determine proinflammatory cytokines TNF- $\alpha$ and IL-1 $\beta$ production in serum of these animals. RT-PCR and Western blot were used to assay the transcriptional level and protein level of TLR4 in cardiac muscle tissue with pathological changes, respectively.

Results: We found that compared with the IR group, ischaemic preconditioning could effectively reduce the expression levels of TNF- $\alpha$ and IL-1 $\beta$ in sera of rats in the IP group $(p<0.01)$. Meanwhile, TLR4 mRNA and protein levels were downregulated ( $p<0.01$ and $p<0.05$, respectively). We also found that infarct size decreased in the IP group compared with the IR group $(p<0.05)$.

Conclusions: Based on the results, we can conclude that the specific mechanism of ischaemic preconditioning for RI might be closely associated with decreasing expression levels of TLR4 and proinflammatory cytokines such as TNF- $\alpha$ and IL-1 $\beta$.

Key words: myocardial ischaemic reperfusion injury, ischaemic preconditioning, TLR4, TNF- $\alpha$, IL-1 $\beta$

\section{Introduction}

Ischaemic heart disease is characterized by reduced blood supply to the cardiac muscle and is the primary cause of death associated with myocardial infarction usually occurring as a result of damage induced by cardiac ischaemia-reperfusion (I/R). Ischaemic preconditioning (IPC) is a phenomenon by which cyclic episodes of brief $I / R$ protect the myocardium against subsequent lethal ischaemic injury. It is known to significantly reduce the infarct size, arrhythmia, and post-ischaemic
Corresponding author: Prof. Zhu Wenzhong, MD, PhD Department of Anesthesia Changhai Hospital

Second Military Medical University

Shanghai 200433, China

E-mail:

zhuwenzhong168@yahoo.com.cn 
contractile dysfunction [1, 2]. Ischaemic preconditioning (IPC) is a potent cellular protective mechanism that is initiated by brief periods of sublethal ischaemic stress. First identified by Murry et al., it has been concluded that the heart adapts within minutes to become resistant against ischaemia-induced injury.

The cellular mechanisms underlying the protection initiated by myocardial IPC have been scrutinized intensively, given the potential to identify novel targets for treating ischaemic heart disease. Work has focused on identifying the triggers, mediators, and end effectors of IPC. Numerous signal transduction pathways have been demonstrated to be mediators affording protection by IPC. Previous studies have demonstrated that phosphatidylinositol 3-kinase (PI3K) and its effector protein kinase B (Akt) are implicated in cell survival and that acute PI3K/Akt activation underlies the infarct size (IS)-limiting effect of ischaemic preconditioning (IP) in the rat heart [3-7]. A recent studies indicated that toll-like receptors (TLRs) are involved in the IP process $[8,9]$.

TLRs represent the first line of host defence against microbial infection and play a pivotal role in both innate and adaptive immunity. TLRs recognize invading pathogens through molecular pattern recognition, transduce signals via distinct intracellular pathways involving a unique set of adaptor proteins and kinases, and ultimately lead to the activation of transcription factors and inflammatory responses. Among 10 TLRs identified in humans, at least two exist in the heart, i.e., TLR2 and TLR4. In addition to the critical role of these in mediating cardiac dysfunction in septic conditions, emerging evidence suggests that the TLRs can also recognize endogenous ligands and may play an important role in modulating cardiomyocyte survival and in ischaemic myocardial injury [9].

The role of TLR4 in ischaemic preconditioning is still unclear; we do not know the change of the expression of TLR4 in the process. In this study we used ischaemic preconditioning models to observe the change of TLR4 expression and the level of proinflammatory cytokines TNF- $\alpha$ and IL-1 $\beta$. Therefore, the purpose of this study was to determine the effect of TLR4 and proinflammatory cytokines TNF- $\alpha$ and IL-1 $\beta$ that is involved in ischaemic preconditioning.

\section{Material and methods}

\section{Myocardial I/R model and IP model protocol}

Eighteen male Sprague-Dawley (SD) rats were randomly separated into sham (sham group), ischaemic reperfusion (IR group) and ischaemic preconditioning (IP group) groups (6/group). The I/R model and the surgical protocol were similar to methods described previously [10-12]. Rats were anaesthetized with urethane anaesthesia $(5 \mathrm{ml} / \mathrm{kg}$ intraperitoneally). Atropine (0.05 mg s.c.) was administered to reduce airway secretions. Animals were orally intubated and connected to a mouse mini-ventilator. Core body temperature was maintained at $37^{\circ} \mathrm{C}$ with a heating pad and monitored with a rectal thermometer.

A median sternotomy was performed, and the left anterior descending coronary artery (LAD) was visualized and completely ligated for $30 \mathrm{~min}$ in I/R mice by tightening an 8-0 silk suture after passing it over a length of PE-10 tubing beneath the LAD at points 1-2 $\mathrm{mm}$ inferior to the left auricle. The 8-0 suture was similarly placed in the sham group but without LAD occlusion. The PE-10 tubing was ligated with the LAD to achieve easier ligature release and better reperfusion. Ischaemia was confirmed visually by the appearance of pale and bulging myocardium in the area at risk (AAR). The ligature was removed, and reperfusion was visually confirmed after 30 min of LAD occlusion. IPC was introduced by three cycles of 5 min ischaemia, followed by 5 min reperfusion, and repeated four times.

\section{Measurement of serum TNF- $\alpha$ and IL-1 $\beta$ level}

Serum TNF- $\alpha$ and IL-1 $\beta$ protein levels were measured by ELISA. Protein was extracted from the heart by pulverization of frozen tissue followed by extraction with lysis buffer containing protease inhibitors. Total protein was determined using a BCA Protein Assay kit (Pierce, Rockford, Illinois).

\section{Western blot analysis}

Tissue samples from the different groups were dissected. Tissues were homogenized in $500 \mathrm{ml}$ of $25 \mathrm{mM}$ Tris- $\mathrm{HCl}$ (pH 7.4) containing 1\% Triton X-100, $0.1 \%$ sodium dodecyl sulfate (SDS), 2 mM EDTA, and $1 \%$ protease inhibitor cocktail (Sigma) and centrifuged at $14,0000 \mathrm{~g}$ for $30 \mathrm{~min}$ at $4^{\circ} \mathrm{C}$. The supernatants were used for Western blot analysis, with specific antibodies detecting TLR4 (Gen Script). Enhanced chemiluminescent (ECL) substrate was from Pierce (Rockford, IL, USA). The polyvinylidene difluoride (PVDF) membrane was from Schleicher and Schuell Co. (Hahnestrasse, Dassel, Germany). According to standard protocols, the blots were probed with primary antibodies, and horseradish peroxidase-conjugated secondary antibodies were used to reveal the specific protein bands with $\mathrm{ECL}$ detection reagents. The intensities of the protein bands were quantified by densitometry.

\section{RT-PCR analysis}

RNA was extracted from fresh rat tissues from the ischaemic area using Trizol (Gibco), 
according to the manufacturer's instructions. RTPCR analysis specific for TLR4 (Forward: 5'-GGACTCTGCCCTGCCACCATTTA-3', Reverse: 5'-CTTGTGC CCTGTGAGGTCGTTGA-3', anticipated size of PCR products $2800 \mathrm{bp}$ ) and $\beta$-actin (Forward: 5'-GTCAGAAGGACTCCTACGTG-3', Reverse: 5'-TAGGAGCCA GGGCAGTAATC-3, anticipated size of PCR products $825 \mathrm{bp}$ ) was then performed using the RT-PCR kit (Takara Biotechnology) according to the manufacturer's instructions.

\section{Determination of myocardial infarct size}

At the end of the perfusion protocols, the coronary artery was re-occluded and $4 \mathrm{ml}$ of $0.1 \%$ Evans blue dye was injected into the aorta to delineate the area at risk. Stained hearts were frozen, sliced, and incubated at $37^{\circ} \mathrm{C}$ in $1 \%$ triphenyltetrazolium chloride to delineate infarcted tissue. Slices were then fixed and quantified. Infarct size was expressed as a percentage of the area at risk. The area at risk was calculated as a percentage of the total ventricular area.

\section{Statistical analysis}

Statistical analysis was done using SPSS 10.0. Parametric data were expressed as mean \pm standard deviation (SD). Comparisons of parameters among the 3 groups were made with one-way ANOVA, followed by the Newman-Keuls test. Comparisons of parameters between 2 groups were made by unpaired Student's $t$ test. A $P$ value less than 0.05 was considered statistically significant.

\section{Results}

\section{Serum level of TNF- $\alpha$ and IL-1 $\beta$}

There were significant $(P<0.01)$ differences observed in the levels of TNF- $\alpha$ and IL-1 $\beta$ between the I/R group and the sham group (Table I), and the levels decreased significantly $(P<0.01)$ in the IP group compared with the I/R group. The results showed that ischaemic preconditioning (IPC) protects the myocardium against lethal ischaemic injury induced by inflammatory factors TNF- $\alpha$ and IL-1 $\beta$.

\section{RT-PCR analysis}

The results (Figure 1) showed that the expression of TLR4 mRNA in the I/R group increased significantly $(p<0.01)$ compared with the sham group, but in the IP group the expression of TLR4 mRNA decreased significantly $(p<0.01)$ compared with the I/R group. The results indicated that ischaemic preconditioning inhibits the expression of TLR4 mRNA.

\section{Western blot analysis}

We found that the expression of TLR4 protein increased significantly $(P<0.01)$ in the I/R group compared with the sham group (Figure 2$)$. There was no significant $(P>0.05)$ difference in the

A

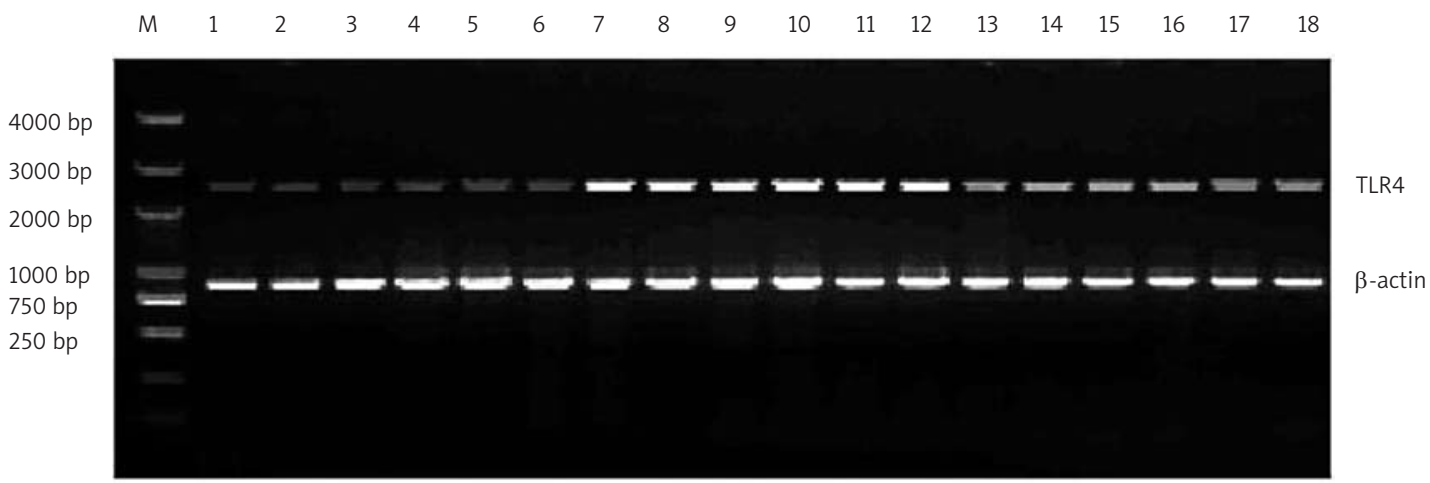

Figure 1. TLR4 mRNA level determined by RT-PCR analysis. A - M: marker; line 1-6: sham group; line 7-12: I/R group; line 13-18: IP group. B - Quantitative results of TLR4 mRNA. RT-PCR analysis for TLR4 and $\beta$-actin in hearts of different groups. $\beta$-actin was used as an internal loading control

${ }^{a} p<0.01$ vs. sham group, ${ }^{b} p<0.01$ vs. $1 / R$ group

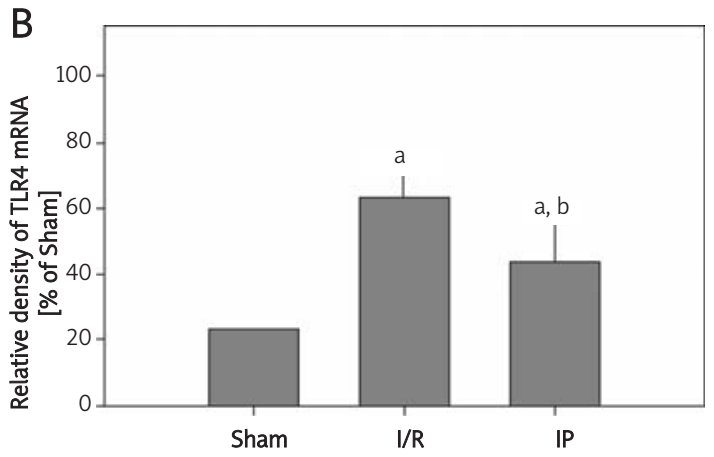


A

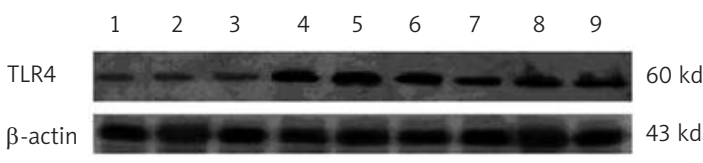

B

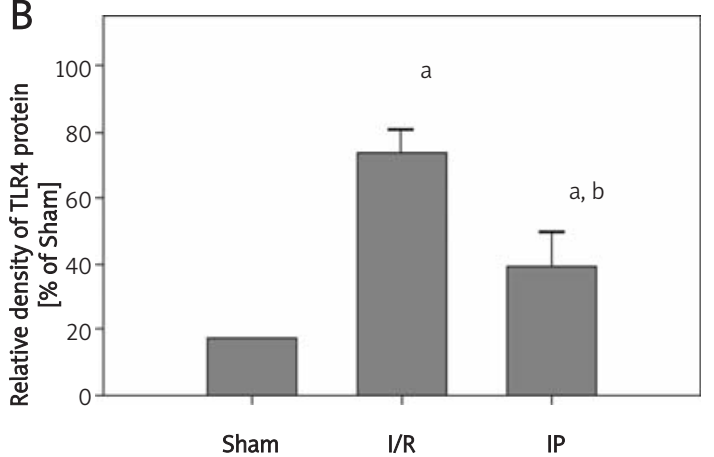

Figure 2. TLR4 protein level determined by Western blot. A- $\beta$-actin was used as an internal control to monitor equal protein sample loading. The expression of TLR4 was markedly increased in the I/R group compared with the sham control group. In the IP group there was attenuated expression of TLR4. B - Quantitative results of TLR4. Mean density of TLR4 in sham-operated group was defined as $100 \%$

$n=6$, mean $\pm S D,{ }^{a} p<0.01 \mathrm{vs}$. sham group, ${ }^{b} p<0.01 \mathrm{vs}$. $1 / R$ group

expression of $\beta$-actin in the 3 groups. When TLR4 protein levels were normalized with the expression of $\beta$-actin protein, the ratio was increased significantly $(P<0.01)$ in the I/R group compared with the sham group, whereas in the IP group the expression of TLR4 protein decreased significantly $(P<0.01)$ compared with the I/R group (Figure 2$)$. These results suggest that TLR4 is activated in I/R, but ischaemic preconditioning inhibits the process.

\section{Determination of myocardial infarct size}

I/R increased the myocardial infarct size. Infarct size in the I/R group was greatly increased compared to the sham group $(P<0.01)$. As shown in Figure 3 , the infarct size in the I/R group is 0.481 \pm 0.089 ; in the IP group $(0.31 \pm 0.029)$ there was a significant decrease of myocardial infarct size versus the I/R group $(P<0.05)$.

\section{Discussion}

In our study, we found that the TLR4 protein level and mRNA level increased in the I/R process; at the

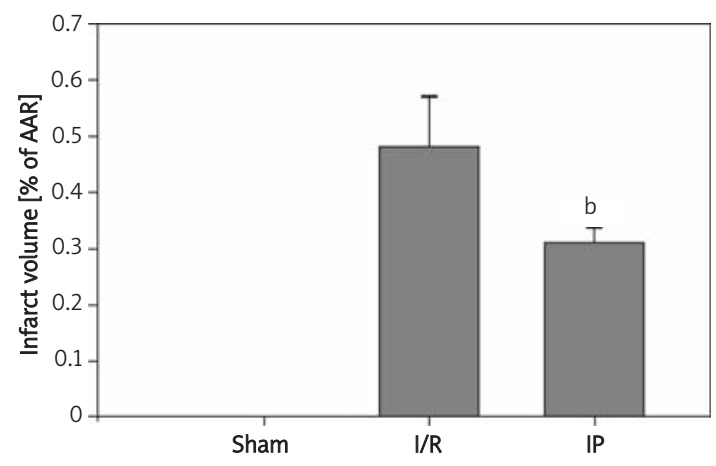

Figure 3. Determination of myocardial infarct size. Infarct size of I/R group is $0.481 \pm 0.089$; in the IP group $(0.31 \pm 0.029)$ there was a significant decrease of myocardial infarct size vs. the I/R group $b P<0.05$ same time TNF- $\alpha$ and IL-1 $1 \beta$ level increased too. As we know, the expression of a constitutively active mutant of human Toll or Toll-like protein in the cell line activated the NF-KB pathway and the expression of $\mathrm{NF}-\mathrm{KB}$-mediated proinflammatory cytokines IL-1, TNF- $\alpha$, and others [13]. We also found that TLR4 and proinflammatory cytokines IL-1 and TNF- $\alpha$ levels decreased in the IP process; these results indicated that the TLR4 signal and inflammation process are involved in the I/R and IP process. In addition, we found a significant decrease of myocardial infarct size in the IP group compared with the I/R group. So we can conclude that TLR4 and proinflammatory factors TNF- $\alpha$ and IL-1 $\beta$ are involved in the IP process. To our knowledge, this is the first study showing that TLR4 and proinflammatory factors TNF- $\alpha$ and IL- $1 \beta$ decreased in the IP process.

Frantz and colleagues first documented that there was enhanced TLR4 expression in remodelling murine myocardium remote from sites of ischaemic injury and in heart tissue from patients with idiopathic dilated cardiomyopathy [14]. In animal models of ischaemia-reperfusion injury or in hypoxic cardiomyocytes in vitro, the administration of a sublethal dose of lipopolysaccharide, which signals through TLR4, reduces subsequent myocardial infarction, improves cardiac functions, and attenuates cardiomyocyte apoptosis $[15,16]$. By contrast, a systemic deficiency of TLR4 leads to attenuated myocardial inflammation, a smaller infarction size, better preserved ventricular function, and reduced ventricular remodelling after ischaemic injury $[17,18]$. These recent studies demonstrate an emerging role for TLRs as a critical modulator in both cell survival and tissue injury in the heart. The precise molecular mechanisms responsible for the phenomenon are still unclear.

In summary, we found that the expression of TLR4 gene and protein decreased in the IP process. The preliminary data need to be well discussed and 
further studies carried out. It is possible that further studies of TLR4 will provide clues for better understanding of its function and may help uncover novel therapeutic strategies for regulating the I/R and IP process.

\section{References}

1. Das DK, Maulik N. Cardiac genomic response following preconditioning stimulus. Cardiovasc Res 2006; 70: 254-63.

2. Shintani-Ishida K, Nakajima M, Uemura K, et al. Ischemic preconditioning protects cardiomyocytes against ischemic injury by inducing GRP78. Biochem Biophys Res Commun 2006; 345: 1600-5.

3. Nagoshi T, Matsui T, Aoyama T, et al. PI3K rescues the detrimental effects of chronic Akt activation in the heart during ischemia/reperfusion injury. J Clin Invest 2005; 115: 2128-38.

4. Maddock HL, Mocanu MM, Yellon DM. Adenosine A(3) receptor activation protects the myocardium from reperfusion/reoxygenation injury. Am J Physiol Heart Circ Physiol 2002; 283: H1307-13.

5 Hausenloy DJ, Yellon DM. New directions for protecting the heart against ischaemia-reperfusion injury: targeting the Reperfusion Injury Salvage Kinase (RISK)-pathway. Cardiovasc Res 2004; 61: 448-60.

6. Schulz R, Cohen MV, Behrends M, Downey JM, Heusch G. Signal transduction of ischemic preconditioning. Cardiovasc Res 2001; 52: 181-98.

7. Heusch G, Boengler K, Schulz R. Cardioprotection: nitric oxide, protein kinases, and mitochondria. Circulation 2008; 118: 1915-9.

8. Chao W. Toll-like receptor signaling: a critical modulator of cell survival and ischemic injury in the heart. Am J Physiol Heart Circ Physiol 2009; 296: H1-12.

9. Valeur HS, Valen G. Innate immunity and myocardial adaptation to ischemia. Basic Res Cardiol 2009; 104: 22-32.

10. Hoffmeyer MR, Jones SP, Ross CR, et al. Myocardial ischemia/reperfusion injury in NADPH oxidase-deficient mice. Circ Res 2000; 87: 812-7.

11. Jones SP, Trocha SD, Strange MB, et al. Leukocyte and endothelial cell adhesion molecules in a chronic murine model of myocardial reperfusion injury. Am J Physiol Heart Circ Physiol 2000; 279: H2196-201.

12. Zhao X, He G, Chen YR, et al. Endothelium-derived nitric oxide regulates postischemic myocardial oxygenation and oxygen consumption by modulation of mitochondrial electron transport. Circulation 2005; 111: 2966-72.

13. Karikó K, Weissman D, Welsh FA. Inhibition of toll-like receptor and cytokine signaling--a unifying theme in ischemic tolerance. J Cereb Blood Flow Metab 2004; 24: 1288-304.

14. Frantz S, Kobzik L, Kim YD, et al. Toll4 (TLR4) expression in cardiac myocytes in normal and failing myocardium. J Clin Invest 1999;104: 271-80.

15. Belosjorow S, Schulz R, Dorge H, Schade FU, Heusch G. Endotoxin and ischemic preconditioning: TNF-alpha concentration and myocardial infarct development in rabbits. Am J Physiol 1999; 277: H2470-5.

16. Belosjorow S, Bolle I, Duschin A, Heusch G, Schulz R. TNF- $\alpha$ antibodies are as effective as ischemic preconditioning in reducing infarct size in rabbits. Am J Physiol Heart Circ Physiol 2003; 284: H927-30.
17. Jun Y, Jian Y, Jia WD, et al. Sequential Expression of TLR4 and its Effects on the Myocardium of Rats with Myocardial Ischemia-Reperfusion Injury. Inflammation 2008; 31: 304-12.

18. Hua F, Ha T, Ma J, et al. Protection against myocardial ischemia/reperfusion injury in TLR4-deficient mice is mediated through a phosphoinositide 3-kinase-dependent mechanism. J Immunol 2007; 178: 7317-24. 